hep-ph/9602315

CPTH-S372.0995

CRETE-95-11

UFIFT-HEP-95-17

Revised June, 1996

\title{
QUANTUM GRAVITY SLOWS INFLATION
}

\author{
N. C. Tsamis* \\ Centre de Physique Théorique, Ecole Polytechnique \\ Palaiseau 91128, FRANCE \\ and \\ Theory Group, FO.R.T.H. \\ Heraklion, Crete 71110, GREECE
}

and

R. P. Woodard ${ }^{\dagger}$

Department of Physics, University of Florida

Gainesville, FL 32611, USA

\begin{abstract}
We consider the quantum gravitational back-reaction on an initially inflating, homogeneous and isotropic universe whose topology is $T^{3} \times \Re$. Although there is no secular effect at one loop, an explicit calculation shows that two-loop processes act to slow the rate of expansion by an amount which becomes non-perturbatively large at late times. By exploiting Feynman's tree theorem we show that all higher loops act in the same sense.
\end{abstract}

\footnotetext{
* e-mail: tsamis@iesl.forth.gr and tsamis@orphee.polytechnique.fr

$\dagger \quad$ e-mail: woodard@phys.ufl.edu
} 


\section{Introduction}

Inflation provides a wonderful explanation for the fact that the cosmic microwave background is observed to be in thermal equilibrium to about one part in $10^{5}$, even for regions of the universe which are only now coming into contact with one another [1]. However, inflationary cosmology has no pretensions of explaining why the dimensionless product of the cosmological constant and Newton's constant is observed to be zero to within about one part in $10^{120}$ [2]. Indeed, the assumption of inflation imposes severe restrictions on any proposal for understanding this grotesque hierarchy. For if we assume that the responsible mechanism can screen a cosmological constant of any size, and that it makes no distinction between the bare cosmological constant and contributions from the matter potential, then one has to explain why inflation was ever able to start. One must also understand why the screening mechanism operates slowly enough to permit the $\approx 55$ e-foldings of inflation needed to produce the isotropy of the cosmic microwave background.

We have proposed that the cosmological constant isn't unreasonably small but only appears so due to the screening effect of infrared processes in quantum gravity [3,4]. * These infrared processes can become strong because the graviton is massless and because a non-zero cosmological constant endows it with a self-interaction of dimension three. (By way of contrast, the massless gluons of QCD possess only dimension four couplings.) Since dimension three couplings between positive norm bosons lower the vacuum energy, the effect is to screen the bare cosmological constant. Like all infrared effects, our process derives from the causal and coherent superposition of interactions throughout the past lightcone. The effect is absent before the onset of inflation because thermal fluctuations

\footnotetext{
* It has also been suggested that screening can occur due to Hawking radiation [5], or from the existence of an infrared fixed point in various effective theories of gravitation [6]. Our mechanism is nearest to Ford's proposal [7], which was based on the assumption that the coincidence limit of the graviton propagator grows in time. Note, however, that our formalism does not show temporal growth for the coincident propagator. It is also significant that - if such growth had been present - Ford's effect would emerge from an entirely different set of diagrams than ours.
} 
disrupt the coherent superposition of interactions from different regions of the very early universe; it is only after inflation has redshifted the temperature that a coherent effect can begin to accumulate. This effect eventually becomes arbitrarily strong because the invariant volume of the past lightcone from the onset of inflation grows without bound as the future unfolds. There is a long period of inflation because an enormous invariant volume is needed to overcome the natural weakness of gravitational interactions.

Since our effect comes from the infrared we can quantize Einstein's theory:

$$
\mathcal{L}=\frac{1}{16 \pi G}(R-2 \Lambda) \sqrt{-g}+(\text { counterterms })
$$

without worrying about the still unknown corrections which must be added to avoid inconsistencies on the Planck scale. The modes which contribute most strongly at any time turn out to have physical wavelengths of about the Hubble radius. As long as the scale of inflation is a few orders of magnitude below the Planck mass, modes which redshift down from the unknown ultraviolet sector will have plenty of time to reach an equilibrium governed by (1).

Another significant feature of our mechanism is its uniqueness to gravity. Interactions mediated by massive quanta cannot give a strong infrared effect because they do not superpose coherently. Conformally invariant quanta cannot give a strong effect because they are insensitive to the enormous invariant volume in the conformally flat geometry created by a long period of homogeneous and isotropic inflation. The graviton is unique among known particles in being massless but not conformally invariant.

A necessary consequence of our mechanism is that asymptotic quantum field theory must break down if one makes the incorrect assumption that the "out" vacuum shows inflation. Previous explicit calculations have confirmed this, both for "in" - "out" matrix elements [3,4], and for scattering amplitudes [8]. These results imply that corrections to the background must become non-perturbatively large at late times, but they do not fix 
the rate at which this occurs. To determine this rate it is necessary to follow the evolution of the expectation value of the background in the presence of a plausible initial state. Our proposal for such a calculation has been discussed elsewhere at great length [4] and we shall content ourselves here with a brief review of the formalism in section 2 . The main point of this paper is to announce that we have brought the calculation to a successful conclusion (section 3 ) and to discuss the result (section 4).

\section{The formalism}

Because it is unlikely for inflation to begin simultaneously over more than a small region, we work on the manifold $T^{3} \times \Re$, with the physical distances of the toroidal radii equal to a Hubble length at the onset of inflation. The object of our study is the expectation value of the invariant element in the presence of a state which is initially free de Sitter vacuum. Since the state is homogeneous and isotropic the result can be expressed in co-moving coordinates:

$$
\left\langle 0\left|g_{\mu \nu}(t, \vec{x}) d x^{\mu} d x^{\nu}\right| 0\right\rangle=-d t^{2}+\mathrm{a}^{2}(t) d \vec{x} \cdot d \vec{x}
$$

We take the onset of inflation to be $t=0$, and we work perturbatively around the classical background:

$$
\mathrm{a}_{\text {class }}(t)=\exp (H t)
$$

where the Hubble constant is $H \equiv \sqrt{\frac{1}{3} \Lambda}$. The actual rate of expansion is given by the effective Hubble constant:

$$
H_{\mathrm{eff}}(t) \equiv \frac{d \ln (\mathrm{a})}{d t}
$$

which is an invariant by virtue of having been defined in a unique coordinate system.

It is simplest to perform the calculation in conformally flat coordinates, for which the invariant element of the background is:

$$
-d t^{2}+\mathrm{a}_{\text {class }}^{2}(t) d \vec{x} \cdot d \vec{x}=\Omega^{2}\left(-d u^{2}+d \vec{x} \cdot d \vec{x}\right)
$$




$$
\Omega \equiv \frac{1}{H u}=\exp (H t)
$$

Note the temporal inversion and the fact that the onset of inflation at $t=0$ corresponds to $u=H^{-1}$. Since the infinite future is at $u=0^{+}$, and since the spatial coordinates fall within the region, $-\frac{1}{2} H^{-1}<x^{i} \leq \frac{1}{2} H^{-1}$, the range of conformal coordinates is rather small. This is why a conformally invariant field - whose dynamics are locally the same as in flat space, except for ultraviolet regularization - cannot induce a big infrared effect.

Perturbation theory is organized most conveniently in terms of a "pseudo-graviton" field, $\psi_{\mu \nu}$, obtained by conformally re-scaling the metric:

$$
g_{\mu \nu} \equiv \Omega^{2} \widetilde{g}_{\mu \nu} \equiv \Omega^{2}\left(\eta_{\mu \nu}+\kappa \psi_{\mu \nu}\right)
$$

As usual, pseudo-graviton indices are raised and lowered with the Lorentz metric, and the loop counting parameter is $\kappa^{2} \equiv 16 \pi G$. After some judicious partial integrations the invariant part of the bare Lagrangian takes the following form [9]:

$$
\begin{array}{r}
\mathcal{L}_{\mathrm{inv}}=\sqrt{-\widetilde{g}} \widetilde{g}^{\alpha \beta} \widetilde{g}^{\rho \sigma} \widetilde{g}^{\mu \nu}\left[\frac{1}{2} \psi_{\alpha \rho, \mu} \psi_{\nu \sigma, \beta}-\frac{1}{2} \psi_{\alpha \beta, \rho} \psi_{\sigma \mu, \nu}+\frac{1}{4} \psi_{\alpha \beta, \rho} \psi_{\mu \nu, \sigma}-\frac{1}{4} \psi_{\alpha \rho, \mu} \psi_{\beta \sigma, \nu}\right] \Omega^{2} \\
-\frac{1}{2} \sqrt{-\widetilde{g}} \widetilde{g}^{\rho \sigma} \widetilde{g}^{\mu \nu} \psi_{\rho \sigma, \mu} \psi_{\nu}^{\alpha}\left(\Omega^{2}\right)_{, \alpha}
\end{array}
$$

Note that each interaction term contains at least one ordinary derivative. This occurs because the dimension three coupling is canceled by the undifferentiated terms from the covariant derivatives of the dimension five coupling. Such a cancellation - for which there is no scalar field or flat space analog — is essential for classical stability [10] against growth of zero modes. An interesting consequence is that the leading infrared effects cancel as well in the quantum theory. However, the two couplings do not agree at subleading order, and there is still a very strong quantum effect.

Gauge fixing is accomplished through the addition of $-\frac{1}{2} \eta^{\mu \nu} F_{\mu} F_{\nu}$ where [9]:

$$
F_{\mu} \equiv\left(\psi_{\mu, \rho}^{\rho}-\frac{1}{2} \psi_{\rho, \mu}^{\rho}+2 \psi_{\mu}^{\rho}(\ln \Omega)_{, \rho}\right) \Omega
$$


The associated ghost Lagrangian is [9]:

$$
\begin{aligned}
& \mathcal{L}_{\text {ghost }}=-\Omega^{2} \bar{\omega}^{\mu, \nu}\left[\widetilde{g}_{\rho \mu} \partial_{\nu}+\widetilde{g}_{\rho \nu} \partial_{\mu}+\widetilde{g}_{\mu \nu, \rho}+2 \widetilde{g}_{\mu \nu}(\ln \Omega)_{, \rho}\right] \omega^{\rho} \\
&+\left(\Omega^{2} \bar{\omega}^{\mu}\right)_{, \mu} \eta^{\rho \sigma}\left[\widetilde{g}_{\nu \rho} \partial_{\sigma}+\frac{1}{2} \widetilde{g}_{\rho \sigma, \nu}+\widetilde{g}_{\rho \sigma}(\ln \Omega)_{, \nu}\right] \omega^{\nu}
\end{aligned}
$$

The zeroth order action results in the following free field expansion [11]:

$$
\psi_{\mu \nu}(u, \vec{x})=\left(\begin{array}{c}
\text { Zero } \\
\text { Modes }
\end{array}\right)+H^{3} \sum_{\lambda, \vec{k} \neq 0}\left\{\Psi_{\mu \nu}(u, \vec{x} ; \vec{k}, \lambda) a(\vec{k}, \lambda)+\Psi_{\mu \nu}^{*}(u, \vec{x} ; \vec{k}, \lambda) a^{\dagger}(\vec{k}, \lambda)\right\}
$$

The spatial polarizations consist of "A" modes:

$$
\Psi_{\mu \nu}(u, \vec{x} ; \vec{k}, \lambda)=\frac{H u}{\sqrt{2 k}}\left(1+\frac{i}{k u}\right) \exp \left[i k\left(u-\frac{1}{H}\right)+i \vec{k} \cdot \vec{x}\right] \epsilon_{\mu \nu}(\vec{k}, \lambda) \quad \forall \lambda \in A
$$

while the space-time and purely temporal polarizations are associated, respectively, with "B" and "C" modes:

$$
\Psi_{\mu \nu}(u, \vec{x} ; \vec{k}, \lambda)=\frac{H u}{\sqrt{2 k}} \exp \left[i k\left(u-\frac{1}{H}\right)+i \vec{k} \cdot \vec{x}\right] \epsilon_{\mu \nu}(\vec{k}, \lambda) \quad \forall \lambda \in B, C
$$

In LSZ reduction one would integrate against and contract into $\Psi_{\mu \nu}(u, \vec{x} ; \vec{k}, \lambda)$ to insert and "in"-coming graviton of momentum $\vec{k}$ and polarization $\lambda$; the conjugate would be used to extract an "out"-going graviton with the same quantum numbers. The zero modes evolve as free particles with time dependences 1 and $u^{3}$ for the A modes, and $u$ and $u^{2}$ for the B and C modes. Since causality decouples the zero modes shortly after the onset of inflation, they play no role in screening and we shall not trouble with them further.

We define $|0\rangle$ as the Heisenberg state annihilated by $a(\vec{k}, \lambda)$ — and the analogous ghost operators - at the onset of inflation. We can use this condition and expansion (10) to express the free pseudo-graviton propagator as a mode sum [8]:

$$
\begin{aligned}
i\left[{ }_{\mu \nu} \Delta_{\rho \sigma}\right]\left(x ; x^{\prime}\right) & \equiv\left\langle 0\left|T\left\{\psi_{\mu \nu}(x) \psi_{\rho \sigma}\left(x^{\prime}\right)\right\}\right| 0\right\rangle_{\text {free }} \\
& =H^{3} \sum_{\lambda, \vec{k} \neq 0}\left\{\theta\left(u^{\prime}-u\right) \Psi_{\mu \nu} \Psi_{\rho \sigma}^{\prime *}+\theta\left(u-u^{\prime}\right) \Psi_{\mu \nu}^{*} \Psi_{\rho \sigma}^{\prime}\right\} e^{-\epsilon\|\vec{k}\|}
\end{aligned}
$$


Note that the convergence factor $e^{-\epsilon\|\vec{k}\|}$ serves as an ultraviolet mode cutoff. Although the resulting regularization is very convenient for this calculation, its failure to respect general coordinate invariance necessitates the use of non-invariant counterterms. These are analogous to the photon mass which must be added to QED when using a momentum cutoff. Just as in QED, these non-invariant counterterms do not affect long distance phenomena.

Because the propagator is only needed for small conformal coordinate separations, $\Delta x \equiv\left\|\vec{x}^{\prime}-\vec{x}\right\|$ and $\Delta u \equiv u^{\prime}-u$, the sum over momenta is well approximated as an integral. When this is done the pseudo-graviton and ghost propagators become [8]:

$$
\begin{aligned}
& i\left[\mu \nu \Delta^{\rho \sigma}\right]\left(x ; x^{\prime}\right) \approx \frac{H^{2}}{8 \pi^{2}}\left\{\frac{2 u^{\prime} u}{\Delta x^{2}-\Delta u^{2}+2 i \epsilon|\Delta u|+\epsilon^{2}}\left[2 \delta_{\mu}^{(\rho} \delta_{\nu}^{\sigma)}-\eta_{\mu \nu} \eta^{\rho \sigma}\right]\right. \\
&\left.-\ln \left[H^{2}\left(\Delta x^{2}-\Delta u^{2}+2 i \epsilon|\Delta u|+\epsilon^{2}\right)\right]\left[2 \bar{\delta}_{\mu}^{(\rho} \bar{\delta}_{\nu}^{\sigma)}-2 \bar{\eta}_{\mu \nu} \bar{\eta}^{\rho \sigma}\right]\right\}(13 \\
& i\left[\mu \Delta_{\nu}\right]\left(x ; x^{\prime}\right) \approx \frac{H^{2}}{8 \pi^{2}}\left\{\frac{2 u^{\prime} u}{\Delta x^{2}-\Delta u^{2}+2 i \epsilon|\Delta u|+\epsilon^{2}} \eta_{\mu \nu}\right. \\
&\left.-\ln \left[H^{2}\left(\Delta x^{2}-\Delta u^{2}+2 i \epsilon|\Delta u|+\epsilon^{2}\right)\right] \bar{\eta}_{\mu \nu}\right\}
\end{aligned}
$$

Parenthesized indices are symmetrized and a bar above a Lorentz metric or a Kronecker delta symbol means that the zero component is projected out, e.g. $\bar{\eta}_{\mu \nu} \equiv \eta_{\mu \nu}+\delta_{\mu}^{0} \delta_{\nu}^{0}$. The decoupling between functional dependence upon spacetime and tensor indices — and the simplicity of each — greatly facilitates calculations.

The conventional Feynman rules give "in"-"out" matrix elements. Schwinger long ago worked out a generalization which gives "in" - "in" expectation values [12]. One first implements forward evolution from the asymptotic past to an arbitrary time $u_{0}$ by the 
functional integral: *

$$
\left.\left\langle\phi\left(u_{0}\right)\right| \text { in }\right\rangle=\left[\left[d \phi^{+}\right] \exp \left(i \int_{u_{0}}^{\infty} d u \mathcal{L}\left[\phi^{+}\right]\right)\right.
$$

One then evolves back to the asymptotic past by means of a different functional integration:

$$
\left\langle\operatorname{in} \mid \phi\left(u_{0}\right)\right\rangle=\left[\left[d \phi^{-}\right] \exp \left(-i \int_{u_{0}}^{\infty} d u \mathcal{L}^{*}\left[\phi^{-}\right]\right)\right.
$$

The generic dummy fields $\phi^{+}$and $\phi^{-}$represent the pseudo-graviton and ghosts, and are required to agree at $u_{0}$. To obtain expectation values in the presence of free vacuum at $u=H^{-1}$ we need only change the upper limits in the action integrals from $\infty$ to $H^{-1}$.

The associated Feynman rules are simple: vertices can either be "+" (conventional) or "-" (conjugated), and propagators can link any two kinds of fields. The three distinct sorts of propagators are:

$$
\begin{gathered}
\left\langle 0\left|T\left\{\psi_{\mu \nu}^{+}(x) \psi_{\rho \sigma}^{+}\left(x^{\prime}\right)\right\}\right| 0\right\rangle_{\text {free }}=H^{3} \sum_{\lambda, \vec{k} \neq 0}\left\{\theta(\Delta u) \Psi_{\mu \nu} \Psi_{\rho \sigma}^{\prime *}+\theta(-\Delta u) \Psi_{\mu \nu}^{*} \Psi_{\rho \sigma}^{\prime}\right\} e^{-\epsilon\|\vec{k}\|} \\
\left\langle 0\left|T\left\{\psi_{\mu \nu}^{+}(x) \psi_{\rho \sigma}^{-}\left(x^{\prime}\right)\right\}\right| 0\right\rangle_{\text {free }}=H^{3} \sum_{\lambda, \vec{k} \neq 0} \Psi_{\mu \nu}^{*} \Psi_{\rho \sigma}^{\prime} e^{-\epsilon\|\vec{k}\|} \\
\left\langle 0\left|T\left\{\psi_{\mu \nu}^{-}(x) \psi_{\rho \sigma}^{-}\left(x^{\prime}\right)\right\}\right| 0\right\rangle_{\text {free }}=H^{3} \sum_{\lambda, \vec{k} \neq 0}\left\{\theta(\Delta u) \Psi_{\mu \nu}^{*} \Psi_{\rho \sigma}^{\prime}+\theta(-\Delta u) \Psi_{\mu \nu} \Psi_{\rho \sigma}^{\prime *}\right\} e^{-\epsilon\|\vec{k}\|}
\end{gathered}
$$

(Recall that $\Delta u \equiv u^{\prime}-u$.) Of course (15a) is just the Feynman propagator (13a), and (15c) is its conjugate. The "mixed" propagator (15b) can be obtained from (13a) by the replacement: $|\Delta u| \longrightarrow-\Delta u$. Because this mixed propagator agrees with (15a) for $\Delta u<0$ and with (15c) for $\Delta u>0$, and because the imaginary part of any propagator is zero for

* The curious integration limits are the result of the temporal inversion which occurs when using the conformal time:

$$
\int_{-\infty}^{t_{0}} d t=-\int_{\infty}^{u_{0}} d u(H u)^{-1}=\int_{u_{0}}^{\infty} d u(H u)^{-1}
$$


spacelike separation, it is simple to show that there will be destructive interference between the various "+" and "-" interaction vertices contributing to a single observation unless all vertices lie within the past lightcone of the observation point.

\section{The result}

We actually computed the amputated expectation value of $\kappa \psi_{\mu \nu}(u, \vec{x})$, then attached the external leg and multiplied by $\Omega^{2}$ to obtain the corrected background. Because the initial condition, the background, and the dynamics are rotationally and translationally invariant, the amputated 1-point function can be expressed in terms of just two functions of $u[4]: *$

$$
D_{\mu \nu}^{\rho \sigma}\left\langle 0\left|\kappa \psi_{\rho \sigma}(x)\right| 0\right\rangle=a(u) \bar{\eta}_{\mu \nu}+c(u) \delta^{0}{ }_{\mu} \delta^{0}{ }_{\nu}
$$

Neither $a(u)$ nor $c(u)$ is independent of the choice of gauge - only the combination of them which goes to make up $H_{\text {eff }}(t)$ is. A necessary condition for $H_{\text {eff }}(t)$ to receive a non-zero contribution at late times $\left(u \longrightarrow 0^{+}\right)$is that either $a(u)$ or $c(u)$ must grow at least as fast as $u^{-4}$ [4]. In fact the only interest is in faster growth, since $u^{-4}$ behavior could be absorbed into a renormalization of $\Lambda$. The fastest growth possible at any order in perturbation theory is powers of $\ln (H u)$ over $u^{4}[4,13]$. These logarithms can come either from integrating the factors of $\Omega=(H u)^{-1}$ one can see on the interaction vertices $(7)$, or from the logarithm term of an undifferentiated propagator (13). The physical origin of the former effect is just the growth of the invariant volume of the past lightcone; the latter effect derives from the increasing correlation of the vacuum at constant invariant separation as inflation proceeds.

The gauged-fixed kinetic operator $D_{\mu \nu}^{\rho \sigma}$ is most conveniently expressed in terms of the kinetic operator $\mathrm{D}_{A} \equiv \Omega\left(\partial^{2}+\frac{2}{u^{2}}\right) \Omega$ for a massless, minimally coupled scalar and the kinetic operator $\mathrm{D}_{B}=\mathrm{D}_{C} \equiv \Omega \partial^{2} \Omega$ for a conformally coupled scalar [9]:

$$
D_{\mu \nu}^{\rho \sigma} \equiv\left[\frac{1}{2} \bar{\delta}_{\mu}^{(\rho} \bar{\delta}_{\nu}^{\sigma)}-\frac{1}{4} \eta_{\mu \nu} \eta^{\rho \sigma}-\frac{1}{2} \delta_{\mu}^{0} \delta_{\nu}^{0} \delta_{0}{ }^{\rho} \delta_{0}{ }^{\sigma}\right] \mathrm{D}_{A}+\delta_{(\mu}{ }^{0} \bar{\delta}_{\nu)}{ }^{(\rho} \delta_{0}{ }^{\sigma)} \mathrm{D}_{B}+\delta_{\mu}{ }^{0} \delta_{\nu}{ }^{0} \delta_{0}{ }^{\rho} \delta_{0}{ }^{\sigma} \mathrm{D}_{C}
$$




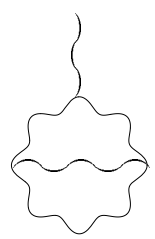

(a)

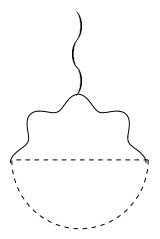

(b)

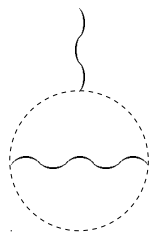

(c)

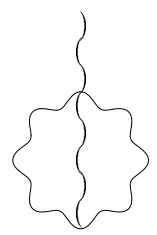

(d)

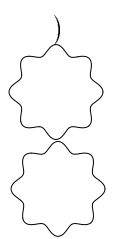

(e)

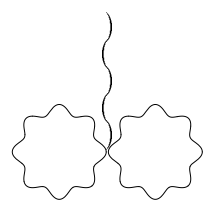

(f)

Fig. 1: Two-loop contributions to the background geometry. Gravitons reside on wavy lines and ghosts on segmented lines.

There are no logarithms at one loop because amputated one-loop tadpoles involve no integrations. In fact, it turns out that the $u^{-4}$ threshold is not even reached. The six diagrams of Fig. 1 contribute to the two-loop tadpole. Diagram (f) can give no logarithms because it involves no integrations, and diagram (e) is entirely canceled by the counterterm needed to renormalize its coincident inner loop. Diagram (d) contributes two logarithms because the volume factor from the integration can be enhanced by the single possible undifferentiated logarithm which can survive from the three propagators. A single undifferentiated logarithm can also survive from the four propagators in diagrams (a-c), and one might think that the two volume factor integrations could be enhanced to produce terms of order $u^{-4} \ln ^{3}(H u)$. However, it turns out that whenever an undifferentiated logarithm survives, the structure of the associated factors always precludes triple logarithms [14]. *

We have reported on the details of the calculation elsewhere [14]. The result is:

$$
a(u)=H^{-2}\left(\frac{\kappa H}{4 \pi u}\right)^{4}\left\{\left(-\frac{1795}{9}+\frac{604}{9}+\frac{320}{3}-\frac{52}{3}\right) \ln ^{2}(H u)+(\text { subleading })\right\}+O\left(\kappa^{6}\right)
$$

Although the reason for this is now clear, it was not obvious from the way in which the calculation had to be broken up in order to be performed efficiently. Indeed, the result did seem to contain triple logarithm terms [15] due to a factor of two error in performing the angular integration for a rare special class of denominators. Now that the result has been corrected and thoroughly checked one can regard the complete cancellation of the triple logarithm terms as a powerful additional check on accuracy. 


$$
c(u)=H^{-2}\left(\frac{\kappa H}{4 \pi u}\right)^{4}\left\{\left(\frac{1157}{3}-\frac{800}{3}-112+8\right) \ln ^{2}(H u)+(\text { subleading })\right\}+O\left(\kappa^{6}\right)
$$

The four numerical coefficients in $a(u)$ and $c(u)$ represent the individual contributions of the diagrams (a) through (d) of Fig. 1 respectively. The external leg is a retarded Green's function in Schwinger's formalism. The procedure for attaching this is straightforward [4], and the invariant interval:

$$
\left\langle 0\left|g_{\mu \nu}(t, \vec{x}) d x^{\mu} d x^{\nu}\right| 0\right\rangle=\Omega^{2}\left\{-[1-C(u)] d u^{2}+[1+A(u)] d \vec{x} \cdot d \vec{x}\right\}
$$

acquires the following leading result for small $u$ :

$$
\begin{gathered}
A(u)=\left(\frac{\kappa H}{4 \pi}\right)^{4}\left\{\left(\frac{7180}{81}-\frac{2416}{81}-\frac{1280}{27}+\frac{208}{27}\right) \ln ^{3}(H u)+(\text { subleading })\right\}+O\left(\kappa^{6}\right) \\
C(u)=\left(\frac{\kappa H}{4 \pi}\right)^{4}\left\{\left(\frac{319}{6}+\frac{49}{3}-52+11\right) \ln ^{2}(H u)+(\text { subleading })\right\}+O\left(\kappa^{6}\right)
\end{gathered}
$$

Comparing this with expressions (2) and (4) gives the following formula for the effective Hubble constant:

$$
\begin{aligned}
H_{\mathrm{eff}}(t) & =\frac{H}{\sqrt{1-C(u)}}\left\{1-\frac{1}{2} u \frac{d}{d u} \ln [1+A(u)]\right\} \\
& =H\left\{1-\left(\frac{\kappa H}{4 \pi}\right)^{4}\left[\left(\frac{4309}{54}-\frac{1649}{27}-\frac{172}{9}+\frac{5}{9}\right)(H t)^{2}+(\text { sub. })\right]+O\left(\kappa^{6}\right)\right\} \\
& =H\left\{1-\left(\frac{\kappa H}{4 \pi}\right)^{4}\left[\frac{1}{6}(H t)^{2}+(\text { subleading })\right]+O\left(\kappa^{6}\right)\right\}
\end{aligned}
$$

Note that each of the four diagrams contributes with the sign we might have expected. The pure graviton diagrams (a) and (d) slow inflation because the negative gravitational interaction energy reduces the vacuum energy. On the contrary, diagrams (b) and (c) have the opposite sign because ghost loops remove unphysical graviton modes from (a) and (d).

It is worth noting that only one other two-loop result has been obtained in quantum gravity, and this was limited to the ultraviolet divergent part for zero cosmological constant [16]. There is little doubt that our formalism is consistent and that the basic reduction procedure is correct, however, it is legitimate to worry about the accuracy of 
implementation in a calculation of this complexity. We have subjected our work to every available check $[14,17]$ and we are reasonably sure it is correct. We nonetheless feel that computer calculations of this scale should be regarded as experiments which can and should be independently verified before being completely trusted.*

\section{Interpretation}

A simple consequence of (19) is that quantum gravity induces an effective stress-tensor which obeys the equation of state for negative vacuum energy, at least in the initial stages of relaxation. To see this we evaluate the classical field equations for the full quantum solution (2) and define the stress tensor as $(8 \pi G)^{-1}$ times the deficit:

$$
G_{\mu \nu}+\Lambda g_{\mu \nu} \equiv 8 \pi G T_{\mu \nu}
$$

The induced energy and pressure can be expressed in terms of $H_{\mathrm{eff}}(t)$ as follows:

$$
\begin{aligned}
& \rho(t)=\frac{6}{\kappa^{2}}\left[H_{\mathrm{eff}}^{2}(t)-H^{2}\right] \\
& p(t)=-\rho(t)-\frac{4}{\kappa^{2}} \dot{H}_{\mathrm{eff}}(t)
\end{aligned}
$$

For (19c) we get:

$$
\rho(t)=-G H^{6}\left\{\left[\frac{1}{8 \pi^{3}}(H t)^{2}+(\text { subdominant })\right]+O\left(\kappa^{2} H^{2}\right)\right\}
$$

where $p=-\rho+$ (subdominant). Note that whenever $H t$ is large enough to compensate for the small prefactor, the subdominant terms are truly insignificant.

The most important consequence of our result is that quantum gravitational processes slow the rate of inflation by an amount which becomes non-perturbatively large at late times. Of course this had to be so in view of the previously cited "in" - "out" results $[3,4,8]$,

\footnotetext{
* We will provide copies of the programs used and the intermediate data generated to anyone who wishes to examine them.
} 
but it is nice to have a proof, and even nicer to know the rate. If there were no further corrections we could estimate the number of e-foldings needed to extinguish inflation as:

$$
H t \sim(\kappa H)^{-2}
$$

For GUT scale inflation this would be about $10^{12}$; the corresponding result for electroweak inflation would be about $10^{68}$. These estimates are not altered by higher loop corrections because the leading contribution at $\ell$ loops has the form:

$$
-\#(\kappa H)^{2 \ell}(\ln (H u))^{\ell}
$$

Of course the number of coupling constants is simple to compute. To get the temporal dependence note that dimensional analysis requires each factor of $\ln (u)$ to be associated either with $\ln (H)$ or $-\ln (\epsilon)$. The factors of $\ln (\epsilon)$ come from ultraviolet divergences, and we know that there can be at most $\ell$ of them in an $\ell$-loop graph. In principle one could get factors of $\ln (H)$ from the upper limits of the conformal time integrations, but dimensional analysis reveals that the integrands fall off too rapidly at large conformal times for this. Therefore, any factor of $\ln (H)$ must come from the logarithm term of an undifferentiated propagator such as (13a) or (13b). But this means that the relevant part of the associated line is just a constant - $\ln (H)$ - so one of the loops is cut and the maximum number of ultraviolet logarithms is one fewer. There can be at most $\ell-1$ undifferentiated logarithms in an $\ell$-loop tadpole, so such a graph can contribute $k$ factors of $\ln (H)$ and up to $\ell-k$ factors of $\ln (\epsilon)$ for $k=0,1, \ldots, \ell-1$. Hence there can be at most $\ell$ factors of $\ln (u)$, and the strongest possible growth at late times is indeed (24).

We can infer the sign of the higher loops effects by using Feynman's tree theorem [18] and appealing to common sense about classical gravitation. The tree theorem is a procedure for decomposing loops into sums of on-shell tree diagrams. The decomposition comes from rearranging the mode expansions of the various propagators (15a-c). For 
example, the "++" propagator can be written as:

$$
\begin{aligned}
& \left\langle 0\left|T\left\{\psi_{\mu \nu}^{+}(x) \psi_{\rho \sigma}^{+}\left(x^{\prime}\right)\right\}\right| 0\right\rangle_{\text {free }}=H^{3} \sum_{\lambda, \vec{k} \neq 0}\left\{\theta(\Delta u) \Psi_{\mu \nu} \Psi_{\rho \sigma}^{\prime *}+\theta(-\Delta u) \Psi_{\mu \nu}^{*} \Psi_{\rho \sigma}^{\prime}\right\} e^{-\epsilon\|\vec{k}\|} \\
& =\theta(\Delta u) H^{3} \sum_{\lambda, \vec{k} \neq 0}\left\{\Psi_{\mu \nu} \Psi_{\rho \sigma}^{\prime *}-\Psi_{\mu \nu}^{*} \Psi_{\rho \sigma}^{\prime}\right\} e^{-\epsilon\|\vec{k}\|}+H^{3} \sum_{\lambda, \vec{k} \neq 0} \Psi_{\mu \nu}^{*} \Psi_{\rho \sigma}^{\prime} e^{-\epsilon\|\vec{k}\|}
\end{aligned}
$$

The first sum is just the retarded propagator, while the second sum is the operator for on-shell propagation from $x^{\prime}$ to $x$. The mixed propagator (15b) is already on-shell, and the "--" propagator (15c) is just the conjugate of (25). Analogous results apply as well for the various ghost propagators. The tree decomposition of a loop is obtained by expanding the various propagators as retarded plus on-shell ones. The term with all retarded propagators vanishes - except for local terms from the singular coincidence limit, which is in the hard ultraviolet and of no concern to us. The remaining terms are sums of on-shell trees.

The tree decomposition allows us to regard deviations from the classical background as the response to an ensemble of on-shell gravitons. (Ghosts cancel against unphysical graviton polarizations as a consequence of decoupling.) This interpretation is advantageous because although isolated classical gravitons carry positive energy, the interaction energy between them is negative, at least for long wavelengths. In fact we have already encountered both terms at lowest order: the positive graviton zero-point energy comprises the one-loop tadpole, while the negative gravitational interaction energy gives the dominant infrared behavior of the two-loop tadpole. Like the one-loop tadpole, the energy of isolated gravitons is ultra-local, ultraviolet divergent and completely subsumed into local counterterms. The gravitational interaction energy is not local because it represents the interaction between diffuse sources. Its pressure obeys the equation of state for vacuum energy, $p=-\rho$, because causality limits the range of interaction to a Hubble radius. The energy density must therefore be independent of the much larger, total volume. Hence the 
total energy is $U=\rho V$ and we have:

$$
p=-\frac{\partial U}{\partial V}=-\rho
$$

We can even understand the prefactor of $G H^{6}$ in (22) by considering the Newtonian gravitational interaction energy due to the zero-point energy of a mode of wavelength $H^{-1}$ confined to a Hubble volume $H^{-3}$.

The time dependence of the effect — which is why it cannot be absorbed into the initial cosmological constant — can be roughly understood by considering the response of the background zero mode to a single graviton of initial momentum $\vec{k} \neq 0$. There is little response initially because the spatial variation of the graviton gives it a weak overlap with the zero mode. Inflation redshifts the physical momentum to $H u \vec{k}$. As the graviton flattens out the zero mode feels it more strongly, however, the process is cut off by causality when the graviton's wavelength redshifts beyond the causal horizon of $H^{-1}$. We expect the maximum effect when:

$$
H u\|\vec{k}\| \sim H \quad \Longrightarrow \quad u \sim\|\vec{k}\|^{-1}
$$

If the radiation ensemble were cut off at high momentum then we would see the ensemble's gravitational interaction energy slow inflation until the shortest wavelength had been redshifted beyond the causal horizon, after which there would be little effect. A locally de Sitter background is stable on the classical level [10] because any classically well defined metric must possess such a cutoff. The fact that quantum gravity continues to show an effect at late times derives from the absence of a cutoff in the tree decomposition, which absence is itself a consequence of the uncertainty principle. We see an ever increasing effect because the number of modes just redshifting past the horizon grows like $\|\vec{k}\|^{2} \sim u^{-2}$. 


\section{Conclusions}

To sum up, we have proposed that inflation happens for no other reason than that the bare cosmological constant is large and positive - there is no need for scalars. The observed universe derives from a patch of about one Hubble volume which began inflation when its local temperature dropped below the scale $M=(H / \kappa)^{1 / 2}$. The reason our universe is not inflating today — at least not rapidly — is that infrared processes in quantum gravity tend to screen the bare cosmological constant. The irresistible force of the effect comes from the fact that it scales as powers of the invariant volume of the past lightcone, which grows without bound as evolution continues. The slowness of the effect derives from the fact that it is a gravitational process, and only the causal and coherent superposition of interactions over an enormous invariant volume can overcome the weakness of the natural coupling constant, $\kappa H \lesssim 10^{-6}$.

All experimentally confirmed matter quanta are either massive or else conformally invariant at the classical level, so they give only negligibly small corrections to the classical geometry of an inflating universe. Although certain conjectured light quanta may be competitive with the graviton for a time, in the end only gravity is left. This means that quantum gravity makes unique predictions for the extinction of inflation. Unfortunately, the most interesting of these are not easy to obtain because they occur after perturbation theory has broken down. In particular, the last sixty e-foldings govern the magnitude and spectrum of observable density perturbations, and the very end of inflation should tell us what reheating temperature was reached.

Without some control of the regime in which the infrared effect becomes large, it is hard to make even qualitative arguments. The onset of the strong effect may be sudden or slow; and the approach of $H_{\text {eff }}(t)$ to zero may be monotonic or involve an "overshoot" followed by damped oscillations between $\Lambda>0$ and $\Lambda<0$ phases. For instance, if the onset is sudden with subsequent oscillations the reheating temperature should be comparable with 
the initial scale of inflation, $\mathrm{T}_{\mathrm{RH}} \sim(H / \kappa)^{1 / 2}$. But if the approach is slow and monotonic we expect a lower value of $\mathrm{T}_{\mathrm{RH}}$.

At least in the perturbative regime, the adiabatic analysis can be shown to underestimate the strength of the effect. For if we divide the time interval into $N$ steps $d t=(t / N)$ and evolve forward by reseting at the beginning of every step the bare cosmological constant to equal its effective value at the end of the previous step, after $N$ iterations we obtain:

$$
H_{\mathrm{eff}}^{(N)}(d t)=H\left\{1-\left(\frac{\kappa H}{4 \pi}\right)^{4}\left[\frac{1}{6} \frac{(H t)^{2}}{N}+(\text { subleading })\right]+O\left(\kappa^{6}\right)\right\}
$$

This result does not agree with the $H_{\text {eff }}(t)$ given by (19c) and gives a weaker effect.

What happens beyond perturbation theory is an open problem. Screening depends upon the strength of the interaction in the past lightcone, the strength of correlations from the past with the present and the accessible volume of the past lightcone. Notice that the first two decline as inflation slows while the third increases. The delicate balance between the different ways the above three factors evolve determines what will actually happen. It is quite possible that the screening is never complete. If so, part of our current expansion is residual inflation, and the time dependence of this component may play a crucial role in resolving, among other things, the apparent problem with the age of the universe. Therefore, there may even be observable effects in the present epoch.

\section{ACKNOWLEDGEMENTS}

We wish to thank T. J. M. Zouros for the use of his SUN SPARC station, and S. Deser for his encouragement and support during this project. We also acknowledge discussions with I. Antoniadis, C. Bachas and J. Iliopoulos. One of us (RPW) thanks the University of Crete and the Theory Group of FO.R.T.H. for their hospitality during the execution of this project. This work was partially supported by DOE contract 86-ER40272, by NSF 
grant 94092715 and by EEC grant 933582 .

\section{REFERENCES}

[1] A. H. Guth, Phys. Rev. D23 (1981) 347.

E. W. Kolb and M. S. Turner, The Early Universe (Addison-Wesley, Redwood City, CA, 1990).

A. D. Linde, Particle Physics and Inflationary Cosmology (Harwood, Chur, Switzerland, 1990).

[2] A. Sandage, Observatory 88 (1968) 91.

[3] N. C. Tsamis and R. P. Woodard, Phys. Lett. B301 (1993) 351.

[4] N. C. Tsamis and R. P. Woodard, Ann. Phys. 238 (1995) 1.

[5] N. P. Myrhvold, Phys. Rev. D28 (1983) 2439.

E. Mottola, Phys. Rev. D31 (1985) 754.

[6] I. Antoniadis and E. Mottola, Phys. Rev. D45 (1992) 2013.

E. Elizalde and S. D. Odintsov, Phys. Lett. B334 (1994) 33.

[7] L. H. Ford, Phys. Rev. D31 (1985) 710.

[8] N. C. Tsamis and R. P. Woodard, Class. Quantum Grav. 11 (1994) 2969.

[9] N. C. Tsamis and R. P. Woodard, Commun. Math. Phys. 162 (1994) 217.

[10] S. Deser and L. F. Abbott, Nucl. Phys. B195 (1982) 76.

P. Ginsparg and M. J. Perry, Nucl. Phys. B222 (1983) 245.

[11] N. C. Tsamis and R. P. Woodard, Phys. Lett B292 (1992) 269.

[12] J. Schwinger, J. Math. Phys. 2 (1961) 407; Particles, Sources and Fields (AddisonWesley, Reading, MA, 1970). 
[13] A. D. Dolgov, M. B. Einhorn and V. I. Zakharov, Phys. Rev. D52 (1995) 717.

[14] N. C. Tsamis and R. P. Woodard, "The Quantum Gravitational Back-Reaction On Inflation," hep-ph/9602310, to appear in Annals of Physics.

[15] R. P. Woodard, in Quantum Infrared Physics, Proceedings of the Workshop at the American University of Paris, 6-10 June 1994, ed. H. M. Fried and B. Müller (World Scientific, Singapore, 1995) pp. 450-459.

[16] M. Goroff and A. Sagnotti, Phys. Lett. B160 (1986) 81; Nucl. Phys. B266 (1986) 709.

[17] N. C. Tsamis and R. P. Woodard, Phys. Rev. D15 (1996) 2621.

[18] R. P. Feynman, Acta Phys. Pol. 24 (1963) 697; in Magic Without Magic, ed. J. Klauder (Freeman, New York, 1972) 355. 